\title{
Serum S100B protein concentrations in SGA/FGR newborns
}

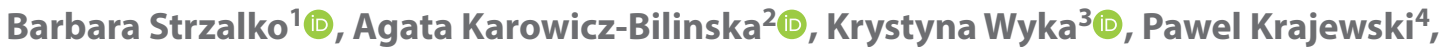 \\ Marcin Kesiak ${ }^{5}$, Bozena Kociszewska-Najman ${ }^{1}[0$ \\ ${ }^{1}$ Department of Neonatology, Children Clinical Hospital, Medical University of Warsaw, Poland \\ ${ }^{2}$ Department of Pathology of Pregnancy, Medical University of Lodz, Regional Specialistic Maternity Hospital, Lodz, Poland \\ ${ }^{3}$ Department of Pediatrics, Oncology, Hematology and Diabetology. Laboratory of Immunopathology and Genetics, \\ Medical University of Lodz, Poland \\ ${ }^{4}$ Division of Neonatology, First Department of Obstetrics and Gynaecology, Medical University of Warsaw, Poland \\ ${ }^{5}$ Department of Neonatology, Regional Specialistic Maternity Hospital, Lodz, Poland
}

\begin{abstract}
Objectives: Fetal growth restriction (FGR) is associated with chronic fetal hypoxia, poor perinatal outcome and increased perinatal mortality. There are no reliable methods to detect cell damage in the central nervous system (CNS) in these patients. The findings of increased an acidic calcium-binding protein (S100B) concentration in biological fluids of infants after brain injury have supported the use of $\mathrm{S100B}$ as a biochemical marker of CNS damage.

The purpose of the study was to assess blood S100B concentrations in small for gestational age (SGA) and appropriate for gestational age (AGA) newborns and to evaluate the usefulness of S100B for early detection of hypoxia.

Material and methods: The investigation was carried out between November 2011 and April 2014. Serum S100B protein level was assessed in cord blood collected from newborns after birth. Medical records of mothers of neonates studied were reviewed for pregnancy induced hypertension $(\mathrm{PIH})$, preeclampsia, maternal smoking during pregnancy and abnormalities in umbilical artery (UA) Doppler ultrasound examination.

Results: The study was carried out in 88 SGA neonates and 80 AGA neonates. The median value of S100B protein concentration in the SGA study group was significantly higher than in AGA controls $(p<0.001)$. Cord blood serum $5100 B$ concentration in SGA neonates with prenatal normal UA Doppler ultrasound findings $(n=32)$ did not differ from that SGA neonates with abnormal prenatal UA Doppler findings $(n=25)(p=0.74)$, but was significantly higher than in AGA newborns $(p<0.001)$.

Conclusions: Elevated S100B protein levels in cord blood collected from SGA newborns may be helpful in detecting infants at higher risk of postnatal neurologic disturbances at an early stage.
\end{abstract}

Key words: S100B protein; biomarker; SGA; FGR, newborn; fetal hypoxia; CNS; neurological damage

Ginekologia Polska 2022; 93, 2: 158-162

\section{INTRODUCTION}

Small for gestational age (SGA) neonates have been defined as those with birthweight below a threshold, the $10^{\text {th }}$ centile. Infants who are SGA may suffer intrauterine fetal growth restriction (FGR). FGR is defined as a persistent suppression of fetal growth potential that occurs in response to a decrease in oxygen and nutrients supply from the mother to the fetus. Clinical evidence suggests that feto-placental insufficiency and fetal pre-exposure to decreased oxygen is associated with chronic fetal hypoxia and increased peri- natal mortality [1]. FGR is associated with poor perinatal outcome, perinatal brain injury and is the strongest risk factor for an unexplained intrauterine death $[2,3]$. About $15 \%$ of infants with FGR develop some degree of neurological damage $[4,5]$. Most SGA/FGR fetuses remain unnoticed until birth, even when routine third-trimester ultrasound is performed [6]. However, not all fetuses measuring less than the $10^{\text {th }}$ percentile for their gestational age are at risk for adverse outcomes and might be constitutionally small. Available diagnostic tools and routine procedures

\section{Corresponding author:}

Barbara Strzałko

Department of Neonatology, Children Clinical Hospital, Medical University of Warsaw, 63A Żwirki i Wigury St, 02-091 Warsaw, Poland

e-mail: strzalko.b@gmail.com 
used when hypoxia is suspected (blood $\mathrm{pH}$, lactate levels, cerebral ultrasound) may not be able to detect cell damage and subclinical lesions in the nervous system. Recently, novel potential biomarkers of brain injury and hypoxia have been identified [7-9]. One of them, S100B is an acidic calcium-binding protein expressed and released by astrocytes and mainly concentrated in the central nervous system. Secretion of $\mathrm{S} 100 \mathrm{~B}$ is an early process of the glial response to metabolic injury (oxygen, serum and glucose deprivation) [9-12]. Previous evidence that $\mathrm{S} 100 \mathrm{~B}$ concentrations are increased in the presence of brain injury suggests that higher S100B levels in pregnancies with FGR reflect fetal chronic hypoxia $[7,13,14]$. Since increased concentration of S100B can easily be found in biological fluids (cerebrospinal fluid, peripheral and cord blood, urine and amniotic fluid), the protein can be used as a biomarker of brain damage in growth restricted newborns [13-18].

The aim of this study was to assess blood S100B concentrations in small for gestational age (SGA) and appropriate for gestational age (AGA) newborns and to evaluate the usefulness of $\mathrm{S100B}$ for early detection of hypoxia. We hypothesized that serum levels of the $\mathrm{S} 100 \mathrm{~B}$ marker might differ in SGA and AGA infants.

\section{MATERIAL AND METHODS}

\section{Patients}

The investigation was performed in a tertiary referral center for neonatal intensive care. All neonates who were born SGA at the Department of Neonatology, Medical University of Łódź, between November 2011 and April 2014 were enrolled in the study.

Birth weight and head circumference in newborns were measured and percentile were assessed. SGA was defined as birth weight $\leq 10^{\text {th }}$ centile. The control group included AGA infants (defined as having birth weight between $10^{\text {th }}$ and $90^{\text {th }}$ centile) delivered consecutively at term. Infants who died at birth, those who had congenital malformations, and children of mothers who refused to participate were excluded. The gestational age of newborns was determined according to the date of the last menstrual period and the first trimester ultrasound exam. Apgar score was determined at five minutes after birth.

SGA newborns were divided according to anthropometric indices into those with symmetrical and those with asymmetrical fetal growth restriction. SGA newborns have been categorized as "asymmetrical" when the birth weight was disproportionally restricted as compared to head circumference and "symmetrical" when both: weight and head circumference were proportionately small. Asymmetrical growth restriction was defined as any birth weight $>1$ SD less than the corresponding head circumference.
Medical records of mothers of neonates studied were reviewed for gestational hypertension (GH), preeclampsia, maternal smoking during pregnancy and abnormalities in umbilical artery (UA) Doppler ultrasound examination. An abnormal pulsatility index PI for UA was defined as above the $95^{\text {th }}$ centile for gestational age for uncomplicated pregnancies.

The study was approved by the local ethics committee (approval No. RNN/175/05/KE). Written informed consent was obtained from all mothers of enrolled neonates.

\section{Blood samples}

Cord blood was obtained from newborns directly after birth. Serum was obtained by centrifugation at $3000 \times g$ for 5 min and stored at $-70^{\circ} \mathrm{C}$ (for up to 6 months) until the biochemical assay.

\section{S100B Assay}

S100B concentrations were quantified by an enzyme-linked immunosorbent assay (ELISA; EIAab Science Co., Wuhan, China), according to the manufacturer's instructions. Samples were analyzed in duplicate and compared with $\mathrm{S100B}$ standard. The lower limit of detection of the ELISA for $\mathrm{S} 100 \mathrm{~B}$ is $1 \mathrm{pg} / \mathrm{mL}$.

\section{Statistical analysis}

Descriptive statistics were used to describe characteristics of the study group. Continuous data were presented as means with standard deviations (SD) or median with minimum and maximum values. Categorical variables were described as frequencies and percentages. The Shapiro-Wilk test was evaluated to determine if the data were normally distributed. Group comparisons for normally distributed data were performed using the $t$ test. When the normality assumptions were not satisfied, Mann-Whitney $U$ test for continuous variables and the Fisher's exact test for binary variables were used. All analyses were performed with the use of IBM SPSS Statistics for Windows V. 25.0.0 (IBM Corp. Armonk, New York, USA). We considered a two-tailed $p$ value less than 0.05 to be significant.

\section{RESULTS}

The study was carried out in 88 SGA neonates and 80 AGA neonates. Birth weight was lower in the SGA group than in the AGA group $(p<0.001)$. There were no significant differences in maternal age, gender characteristics, mode of delivery and cord blood lactate concentration between the SGA and AGA groups. There was statistically significant difference in the Apgar score and gestational age (lower in SGA group) (Tab.1). All SGA infants were above or equal 32 weeks of gestation. 
None of the newborns had chromosomal abnormalities or TORCH infection. In the SGA group, maternal smoking during pregnancy, gestational hypertension $(\mathrm{GH})$ and preeclampsia were observed significantly more often than in the AGA group (Tab. 1). Absent end-diastolic flow in UA was found in 13 cases of SGA group with abnormal prenatal Doppler.

Cord blood serum concentration of S100B protein at birth was significantly higher in the SGA infants than in the AGA group ( $<<0.001)$ (Tab. 2).

UA Doppler examination was performed in 57 out of $88(65 \%)$ mothers of newborns from the study group. Cord blood serum S100B concentration in SGA neonates with prenatal normal UA Doppler ultrasound findings $(n=32$, median 69.3 [0.3-463.5]) did not differ from that SGA neonates with abnormal prenatal UA Doppler findings $(n=25$, median 44.4 [6.8-1110.2]) ( $p=0.74$ ) but was significantly higher than in AGA newborns $(n=80)(p<0.001)(T a b .2)$.
S100B concentration was significantly higher in both SGA with symmetrical $(n=29)$ and asymmetrical $(n=59)$ fetal growth restriction than in non-growth restricted AGA neonates ( $p<0.001)$ (Tab. 2).

Ultrasound examination of central nervous system was performed in all SGA infants within first 48 hours of life. Four out of 88 SGA infants had abnormal Rl in the anterior cerebral artery $(<0.6)$ in the first head ultrasound examination.

We found no statistically significant differences in $\mathrm{S} 100 \mathrm{~B}$ concentration in terms of the anterior cerebral artery Doppler scanning between SGA newborns with abnormal RI (<0.6) and normal RI (>0.6).

\section{DISCUSSION}

The study showed that serum $\mathrm{S} 100 \mathrm{~B}$ concentrations in the SGA infants were higher than in the AGA group. Our findings confirm the previous results of Gazzolo et al. [13], who studied S100B levels in cord blood from FGR new-

\begin{tabular}{|c|c|c|c|}
\hline Characteristic & SGA group & AGA group & p-value \\
\hline Male neonates, n (\%) & $88(52)$ & $80(56)$ & 0.64 \\
\hline Mean birth weight, g (SD) & $2132(543)$ & $3491(366)$ & 0.001 \\
\hline Mean gestation, weeks (SD) & $37.1(3.0)$ & $39.1(1.0)$ & $<0.001$ \\
\hline Median Apgar score at $5 \min (\min -\max )$ & $10(5-10)$ & $10(8-10)$ & $<0.001$ \\
\hline Cord blood lactate, mg/dL (SD) & $45.61(16.1)$ & $45.6(14.7)$ & 0.99 \\
\hline Mean maternal age, years (SD) & $31.1(5.0)$ & $30.0(4.6)$ & 0.15 \\
\hline Mode of delivery, $\mathrm{n}(\%)$ & & & 0.13 \\
\hline Spontaneous VD & $20(23)$ & $33(41)$ & \\
\hline Operative VD & $1(1)$ & 0 & \\
\hline Cesarean section & $67(76)$ & $47(59)$ & \\
\hline Smoking during pregnancy, $\mathrm{n}(\%)$ & $20(23)$ & $4(5)$ & 0.002 \\
\hline $\mathrm{GH}, \mathrm{n}(\%)$ & $22(25)$ & $3(4)$ & $<0.001$ \\
\hline Preeclampsia, n (\%) & $5(6)$ & 0 & 0.06 \\
\hline \multicolumn{4}{|l|}{ Antenatal Doppler changes: } \\
\hline $\mathrm{PI}>95^{\text {th }}$ centile in UA, $\mathrm{n}(\%)$ & $25(28)$ & $2(3)$ & $<0.001$ \\
\hline Absent end-diastolic flow in UA, $n$ (\%) & $13(15)$ & & \\
\hline
\end{tabular}

The bold $\mathrm{p}$-values highlight whether the differences observed were statistically significant. SD — standard deviation; SGA — small for gestational age; AGA — appropriate for gestational age; $\mathrm{GH}$ - gestational hypertension; $\mathrm{UA}$ — umbilical artery; $\mathrm{VD}$ — vaginal delivery

Table 2. Cord blood S100B concentrations in AGA, all SGA, those SGA that had normal UA PI and SGA newborns with symmetrical growth

\begin{tabular}{|l|l|l|l|l|l|}
\hline & Group & $\mathbf{n}$ & Median & Min-max \\
\hline & AGA + & 80 & 25.7 & $0.1-395.8$ \\
\hline \multirow{2}{*}{ S100B $(\mathrm{pg} / \mathrm{mL})$} & SGA & 88 & 64.3 & $0.1-2025.0$ \\
\cline { 2 - 5 } & SGA with normal UA PI & 32 & 69.3 & $0.3-463.5$ & $<0.001$ \\
\cline { 2 - 4 } & "Symmetrical" SGA & 29 & 58.5 & $0.3-95.9$ & $<0.001$ \\
\hline
\end{tabular}

The bold $p$-values highlight whether the differences observed were statistically significant. AGA group serves as a reference ( $t$ ) when compared separately with the SGA group and the SGA with normal UA PI. AGA — appropriate for gestational age; SGA — small for gestational age; UA — umbilical artery; PI — pulsatility index 
born delivered by elective caesarean section and published the first paper about S100B protein and FGR correlation in 2002. In this study higher S100B levels in umbilical blood of FGR newborn were demonstrated.

Since $\mathrm{S} 100 \mathrm{~B}$ protein, during an active brain injury, is released from a damaged tissue into circulation, its concentration increases at an early stage of hypoxia in both cerebrospinal fluid and cord blood. The best sources for biomarkers are fluids obtained the least invasively and shortly after birth [8]. As $\mathrm{S} 100 \mathrm{~B}$ protein is highly concentrated in central nervous system, and has a half-life about one hour, is released by kidney tissue and increases in biological fluids at an early stage. Cord blood and urine seems to be a perfect source for further studies of the potential use of $\mathrm{S100B}$ measurements in FGR pregnancies and newborns. Gazzolo et al. [19], reported that higher concentrations of $\mathrm{S} 100 \mathrm{~B}$ were detected in cord blood of FGR fetuses who developed intraventricular hemorrhage after birth. In addition, the authors observed that the highest maternal S100B concentrations were found in group of fetuses with prenatal brain-sparing effect in prenatal ultrasound examination. In another study, Florio et al., found higher $\mathrm{S} 100 \mathrm{~B}$ concentrations in urine samples taken shortly after birth in FGR newborns compared to matched AGA controls. They noticed highest S100B concentrations in the neonates with abnormal neurologic follow-up but also significantly high in FGR infants with uneventful neurologic follow-up at one week of age [15]. However, no differences in S100B serum concentration of FGR and AGA infants were found in a small study containing 20 infants in each group [16]. In another study, Gazzolo et al. [13], found a correlation between circulating S100B protein and the fetal middle cerebral artery pulsatility index (MCA PI) that might suggest cerebral cell damage in growth restricted fetuses. This study showed no difference in S100B levels between SGA newborns with no abnormalities in prenatal Doppler examination (normal UA PI) and neonates with normal growth (AGA).

In our study, we attempted to investigate whether S100B concentration depends on prenatal hemodynamic disturbances. When SGA newborns were grouped according to normal and abnormal prenatal UA Doppler examination (UA PI $>95^{\text {th }}$ centile), we did not find any differences in serum S100B concentration between either group. In contrast to Gazzolo et al. [13], we observed significantly higher S100B concentrations in SGA newborns with normal Doppler examination compared to AGA newborns. However, the prenatal ultrasound examinations were not performed in all cases and thus these results should be interpreted with caution.

In this study we also divided SGA group according to the traditional biometric birth measurements to those with symmetrical and asymmetrical growth restriction. Factors that cause symmetric growth restriction tend to develop early during fetal life. Since all SGA newborns studied were older than 32 weeks of gestation, we could speculate that beside growth restricted newborns, "symmetrical SGA" group included constitutionally and physiologically small infants. Both"symmetrical" and "asymmetrical" neonates had higher cord blood S100B concentrations compared to AGA neonates but these non-growth restricted infants could not be recognized on a group level.

The importance of biometric as well as functional parameters for identification of FGR was reported by Baschat [20], then the consensus-based definition of FGR was established in the Delphi criteria [21]. In our study we found that assessment of S100B concentration may be helpful in identifying FGR while Doppler findings are normal. Further studies however are needed to confirm our results.

\section{CONCLUSIONS}

Increased S100B protein concentration in blood collected from SGA newborns indicates secretion of this marker in response to hypoxia occurring in intrauterine growth restriction. Examination of cord blood $\mathrm{S} 100 \mathrm{~B}$ concentration may be helpful in identifying SGA newborns at a higher risk of postnatal neurological sequelae at an early stage while prenatal Doppler examination is normal, standard clinical and laboratory parameters are silent, and an early-stage neurologic follow-up is uneventful.

\section{Acknowledgements}

Author are indebted to Robert Foltyn, MSc, and Przemyslaw T. Paradowski, MD, PhD, for assistance in preparing the manuscript.

\section{Funding}

The work was supported by grant no 503/1-004-04/ 50311-001 from the Medical University in Łódź, Poland.

\section{Conflict of interests}

The authors declare that they have no competing interests.

\section{REFERENCES}

1. Romo A, Carceller R, Tobajas J. Intrauterine growth retardation (IUGR) epidemiology and etiology. Pediatr Endocrinol Rev. 2009; 6 Suppl 3: 332-336, indexed in Pubmed: 19404231.

2. Gardosi J, Kady SM, McGeown P, et al. Classification of stillbirth by relevant condition at death (ReCoDe): population based cohort study. BMJ. 2005; 331(7525): 1113-1117, doi: 10.1136/bmj.38629.587639.7C, indexed in Pubmed: 16236774

3. Frøen JF, Gardosi JO, Thurmann A, et al. Restricted fetal growth in sudden intrauterine unexplained death. Acta Obstet Gynecol Scand. 2004; 83(9): 801-807, doi: 10.1111/j.0001-6349.2004.00602.x, indexed in Pubmed: 15315590.

4. Rees S, Harding R. Brain development during fetal life: influences of the intra-uterine environment. Neurosci Lett. 2004; 361(1-3): 111-114, doi: 10.1016/j.neulet.2004.02.002, indexed in Pubmed: 15135906.

5. Pallotto EK, Kilbride HW. Perinatal outcome and later implications of intrauterine growth restriction. Clin Obstet Gynecol. 2006; 49(2): 257-269, doi: 10.1097/00003081-200606000-00008, indexed in Pubmed: 16721105. 
6. Figueras $\mathrm{F}$, Eixarch $\mathrm{E}$, Gratacos $\mathrm{E}$, et al. Predictiveness of antenatal umbilical artery Doppler for adverse pregnancy outcome in small-for-gestational-age babies according to customised birthweight centiles: population-based study. BJOG. 2008; 115(5): 590-594, doi: 10.1111/j.1 471-0528.2008.01670.x, indexed in Pubmed: 18333939.

7. Gazzolo D, Abella R, Marinoni E, et al. Circulating biochemical markers of brain damage in infants complicated by ischemia reperfusion injury. Cardiovasc Hematol Agents Med Chem. 2009; 7(2): 108-126, doi: 10.2174/187152509787847119, indexed in Pubmed: 19355873.

8. Florio $\mathrm{P}$, Abella $\mathrm{R}$, Marinoni $\mathrm{E}$, et al. Biochemical markers of perinatal brain damage. Front Biosci (Schol Ed). 2010; 2: 47-72, doi: 10.2741/s45, indexed in Pubmed: 20036928.

9. Douglas-Escobar M, Weiss MD. Biomarkers of hypoxic-ischemic encephalopathy in newborns. Front Neurol. 2012; 3: 144, doi: 10.3389/fneur.2012.00144, indexed in Pubmed: 23130015.

10. Michetti F, Gazzolo D. S100B protein in biological fluids: a tool for perinatal medicine. Clin Chem. 2002; 48(12): 2097-2104, indexed in Pubmed: 12446464.

11. Filippidis AS, Papadopoulos DC, Kapsalaki EZ, et al. Role of the S100B serum biomarker in the treatment of children suffering from mild traumatic brain injury. Neurosurg Focus. 2010; 29(5): E2, doi: 10.3171/2010.8.FOCUS10185, indexed in Pubmed: 21039136.

12. Gerlach $R$, Demel $G$, König HG, et al. Active secretion of $\mathrm{S} 100 \mathrm{~B}$ from astrocytes during metabolic stress. Neuroscience. 2006; 141(4): 1697-1701, doi: 10.1016/j.neuroscience.2006.05.008, indexed in Pubmed: 16782283.

13. Gazzolo D, Marinoni E, di lorio $\mathrm{R}$, et al. Circulating S100beta protein is increased in intrauterine growth-retarded fetuses. Pediatr Res. 2002; 51(2): 215-219, doi: 10.1203/00006450-200202000-00015, indexed in Pubmed: 11809917.

14. Tskitishvili E, Komoto $Y$, Temma-Asano K, et al. S100B protein expression in the amnion and amniotic fluid in pregnancies compli- cated by pre-eclampsia. Mol Hum Reprod. 2006; 12(12): 755-761, doi: 10.1093/molehr/gal083, indexed in Pubmed: 17023485.

15. Florio $\mathrm{P}$, Marinoni $\mathrm{E}$, Di lorio $\mathrm{R}$, et al. Urinary $\mathrm{S} 100 \mathrm{~B}$ protein concentrations are increased in intrauterine growth-retarded newborns. Pediatrics. 2006; 118(3): e747-e754, doi: 10.1542/peds.2005-2875, indexed in Pubmed: 16923924.

16. Boutsikou T, Mastorakos G, Kyriakakou M, et al. Circulating levels of inflammatory markers in intrauterine growth restriction. Mediators Inflamm. 2010; 2010: 790605, doi: 10.1155/2010/790605, indexed in Pubmed: 20614004.

17. Liu Li, Zheng CX, Peng SF, et al. Evaluation of urinary S100B protein level and lactate/creatinine ratio for early diagnosis and prognostic prediction of neonatal hypoxic-ischemic encephalopathy. Neonatology. 2010; 97(1): 41-44, doi: 10.1159/000227292, indexed in Pubmed: 19590245.

18. Velipaşaoğlu M, Yurdakök M, Özyüncü Ö, et al. Neural injury markers to predict neonatal complications in intrauterine growth restriction. J Obstet Gynaecol. 2015; 35(6):555-560, doi: 10.3109/01443615.2014.978848, indexed in Pubmed: 25392968.

19. Gazzolo D, Marinoni E, Di lorio R, et al. High maternal blood S100B concentrations in pregnancies complicated by intrauterine growth restriction and intraventricular hemorrhage. Clin Chem. 2006; 52(5): 819-826, doi: 10.1373/clinchem.2005.060665, indexed in Pubmed: 16543391.

20. Baschat AA. Neurodevelopment following fetal growth restriction and its relationship with antepartum parameters of placental dysfunction. Ultrasound Obstet Gynecol. 2011;37(5):501-514, doi: 10.1002/uog.9008, indexed in Pubmed: 21520312.

21. Gordijn SJ, Beune IM, Thilaganathan B, et al. Consensus definition of fetal growth restriction: a Delphi procedure. Ultrasound Obstet Gynecol. 2016; 48(3): 333-339, doi: 10.1002/uog.15884, indexed in Pubmed: 26909664 